\title{
Is atomised intranasal cocaine systemically absorbed during endoscopic sinus surgery?*
}

\author{
Dean E. Page', Joanne Rimmer 1,2, Michael Keane', Shashi Manikappa', \\ Danielle Butzbach ${ }^{3}$, Charles Giddings ${ }^{1,2}$ \\ Rhinology 57: 3, 200 - 205, 2019 \\ https://doi.org/10.4193/Rhin18.207 \\ 'Monash Health, Melbourne, Australia \\ *Received for publication: \\ 2 Monash University, Melbourne, Australia \\ September 2, 2018 \\ ${ }^{3}$ Forensic Science, South Australia, Australia \\ Accepted: December 4, 2018
}

Background: In order to perform endoscopic sinus surgery (ESS) safely and efficiently, preparation of the nasal mucosa with vasoconstrictor agents is crucial to minimise bleeding. There is no single best method, although traditionally cocaine has been the agent of choice. However, there have been concerns over the potential for systemic side effects when applied topically. With this concern in mind, there exists limited contemporary research looking at cocaine absorption in the context of ESS. This study aims to determine the amount and duration of systemic cocaine absorption after intranasal atomised administration of modified Moffett's solution prior to ESS.

Methodology: Twelve adults undergoing ESS were enrolled. Modified Moffett's solution containing 100 mg cocaine hydrochloride and $1 \mathrm{mg}$ of adrenaline was administered topically 5 minutes prior to surgery. Serum cocaine and the cocaine degradation product, benzoylecgonine, were measured at specific time points up to $12 \mathrm{hrs}$ post administration.

Results: Peak plasma cocaine concentration occurred between 60 and 120 minutes post-administration (range 13-31 $\mu \mathrm{g} / \mathrm{L}$ ). The mean peak plasma concentration was $20.35 \mu \mathrm{g} / \mathrm{L}$ occurring at 120 minutes post-administration. Benzoylecgonine was detected in 11 subjects at 60 minutes post-administration and in all subjects 12 hours post-administration of cocaine.

Conclusions: This study demonstrates that there are low levels of systemic absorption of cocaine when administered in an atomised modified Moffett's formulation prior to endoscopic sinus surgery.

Key words: cocaine, nasal absorption, nasal surgical procedures, paranasal sinus disease, surgical blood loss

\section{Introduction}

In order to perform endoscopic sinus surgery (ESS) safely and efficiently, meticulous preparation of the nasal mucosa with vasoconstrictor agents is crucial to minimise bleeding. Several topical preparation methods are currently used including co-phenylcaine (lignocaine and phenylephrine), adrenaline (various concentrations) and cocaine-containing solutions including Moffett's and its modified version without the sodium bicarbonate buffer. These drugs are applied in various methods including sprays, atomisers, pastes, and using cotton pledgets. There is no single best method and the decision is often at the surgeon's discretion, however traditionally cocaine has been the agent of choice ${ }^{(1,2)}$. Its effects on the nasal mucosa include anaesthesia by way of sodium channel blockade and strong vasoconstriction by preventing noradrenaline reuptake ${ }^{(3)}$. Although still used in many centres, there have been concerns over the potential for systemic side effects even when only applied topically. Systemic side effects may include myocardial ischaemia and arrhythmias, hypertension, hyperthermia, seizures and death.

Studies have confirmed that cocaine may be absorbed through the nasal mucosa, with blood concentrations depending on the amount applied, method of application and time in contact with the mucosa ${ }^{(4-6)}$. Approximately $35 \%$ of a $4 \%$ cocaine solution may be absorbed when applied on cotton pledgets ${ }^{(4,5)}$. However, when Moffett's solution containing cocaine and adrenaline was applied this gave rise to a low mean plasma peak concentration 
with potentially only $5 \%$ absorbed ${ }^{(6)}$. There may be rapid systemic absorption, occurring within 10 minutes, however, other studies demonstrate a slower response ${ }^{(5-8)}$.

There remains limited information on the total duration cocaine may remain in the plasma after topical application to the nasal mucosa. A study by Van Dyke et al demonstrated measurable plasma cocaine levels up to $6 \mathrm{hrs}$ after a $10 \%$ solution (total dose $1.5 \mathrm{mg} / \mathrm{kg}$ ) was applied topically to the nasal mucosa prior to nasal intubation ${ }^{(7)}$.

Overall there exists limited contemporary research looking at cocaine absorption in the context of ESS. No studies have looked specifically at modified Moffett's solution, particularly in an atomised application method. This study aims to determine the amount and duration of systemic cocaine absorption after intranasal atomised administration of modified Moffett's solution prior to ESS.

\section{Material and Methods}

Ethics approval was granted by the Monash Health Human Research and Ethics Committee (reference LNR/16/MonH/343).

\section{Patient recruitment}

Adult patients ( $>18$ years) undergoing ESS were recruited. Patients were excluded if they admitted to cocaine use within 2 weeks of surgery, cocaine application was contraindicated (sensitivity to cocaine, significant ischaemic heart disease (IHD), history of arrhythmia, pregnancy or breastfeeding), or there was the potential for limited mucosal application of solution (grade 3 polyps in 1 or more nasal cavities, obstructing intranasal mass or severe septal deformity).

\section{Modified Moffett's application}

Modified Moffett's solution was prepared by the investigator as per the Monash Health protocol comprising $2 \mathrm{ml}$ of $5 \%$ cocaine hydrochloride $(100 \mathrm{mg}$ ) and $1 \mathrm{ml}$ of 1:1000 adrenaline (1 $\mathrm{mg}$ ) made up to $10 \mathrm{ml}$ with $0.9 \%$ normal saline. Sodium bicarbonate is difficult to attain in small volumes and less routinely used across Australia thus was not included in the solution.

Enrolled patients were intubated under routine general anaesthesia with a throat pack in situ. An additional intravenous cannula (IV) was inserted for blood collection. Each patient was positioned in a 30 degree head down position prior to $5 \mathrm{mls}$ of Modified Moffett's being atomised into each nostril using MAD NasalTM device (product patent \#6.112,743) by the operating surgeon. After 5 minutes the patient was placed into a 30 degree head up position prior to the commencement of surgery. Residual solution was suctioned from the postnasal space at the start of the procedure. If additional haemostasis was required intraoperatively, cotton pledgets containing 1:10,000 adrenaline solution were used.

\section{Serum cocaine and metabolite levels}

All blood samples were taken from the IV cannula inserted after induction. A baseline blood sample to check for pre-existing serum cocaine was taken 10 minutes prior to administration of the modified Moffett's solution. When the modified Moffett's solution was administered this was deemed as time 0 and blood samples were subsequently collected at 10 minutes, 20 minutes, 30 minutes, 60 minutes, 2 hours, 4 hours, 6 hours and 12 hours post-administration of the modified Moffett's solution. Blood samples were immediately stored at $-20^{\circ} \mathrm{C}$ and subsequently transported to $\mathrm{a}-80^{\circ} \mathrm{C}$ fridge for further storage prior to testing. All blood samples were tested by the Forensic Science South Australia laboratory for cocaine and its principal degradation product, benzoylecgonine. The test assay was validated for the quantitation of cocaine and benzoylecgonine in blood from 0.005-0.5 mg/L and 0.01-1.0 mg/L, respectively. The limits of detection were $0.002 \mathrm{mg} / \mathrm{L}$ for cocaine and $0.006 \mathrm{mg} / \mathrm{L}$ for benzoylecgonine. The cocaine and benzoylecgonine were extracted from whole blood $(0.20 \mathrm{~mL})$ using mixed mode solid-phase extraction (SPE). The analytes were protonated by the addition of a pH 5.7 acetate buffer and are retained on the SPE cartridge by cation exchange. Analyte elution was achieved by passing a basified mixture of dichloromethane/iso-propanol (80:20) through the SPE cartridge. The eluent was evaporated and the residue reconstituted into $0.1 \%$ formic acid in water. The extract was analysed by liquid chromatography mass spectrometry (LC-MS/MS) using an electrospray ionisation source (ESI) and triple quadrupole detector. Deuterated analogues of cocaine and benzoylecgonine are used as internal standards to ensure accurate recoveries.

A blank and quality control sample were included with each batch run. Linear calibration curves were constructed with $1 / x$ weighting and used to calculate the cocaine and benzoylecgonine concentrations in the samples, which were analysed in duplicate with the mean reported.

\section{Results}

Twelve adult patients undergoing ESS were enrolled in the study. The demographic and medical information is presented in Table 1. The mean age was 50 years (range 25-79) and the mean body mass index (BMI) was 28 (range 21-34). No patients had documented renal or liver disease. Subject 1 and 8 admitted to intermittent topical decongestant use prior to surgery. Subject 6 had received oral steroids several weeks prior to surgery. No patients had cocaine or its degradation product, benzoylecgonine, in the system prior to surgery. Systemic cocaine concentrations are presented in Table 2 and Figure 1. Six patients demonstrated a peak concentration at 120 minutes post admi- 
Table 1. Demographics and medical information.

\begin{tabular}{|cccc|}
\hline Subject & Age & BMI & Relevant medications \\
\hline 1 & 64 & 30 & Decongestant \\
\hline 2 & 34 & 25 & \\
\hline 3 & 63 & 32 & \\
\hline 4 & 43 & 27 & \\
\hline 5 & 30 & 30 & Prednisolone \\
\hline 6 & 79 & 23 & \\
\hline 7 & 29 & 21 & \\
\hline 8 & 60 & 29 & \\
\hline 9 & 48 & 23 & \\
\hline 10 & 25 & 25 & \\
\hline 11 & 57 & 34 & \\
\hline 12 & 73 & 33 & \\
\hline Mean & 50 & 28 & \\
\hline & & & \\
\hline
\end{tabular}

nistration (range 13-30 $\mu \mathrm{g} / \mathrm{L}$ ). Five patients had an earlier peak at 60 minutes (range 14-31 $\mu \mathrm{g} / \mathrm{L}$ ). Only participant 6 demonstrated a late peak $(42 \mu \mathrm{g} / \mathrm{L})$ at 240 minutes post administration. The mean plasma concentration observed at the most common time to peak (120 mins) was $20.35 \mu \mathrm{g} / \mathrm{L}$ (Table 3). The mean peak concentration across all participants was $22.6 \mu \mathrm{g} / \mathrm{L}$.

Cocaine levels decreased steadily from the peak concentration and were detectable up to 6 hours post-administration but eliminated by 12 hours post-administration.

Plasma concentrations of benzoylecgonine, the principal degradation product of cocaine, are presented in Table 4 and Figure 2. Benzoylecgonine was first detected in 11 subjects at 60 minutes post-administration and in one earlier at 30 minutes post-administration. The earlier detection of benzoylecgonine in subject 11 correlated with a higher earlier concentration of systemic cocaine. Peak levels were detected at two to six hours post-administration, with a mean peak level of $81 \mu \mathrm{g} / \mathrm{L}$ seen after six hours (Table 4). Benzoylecgonine was detected in all subjects 12 hours post-administration of cocaine, with a mean level of $51.7 \mu \mathrm{g} / \mathrm{L}$.

\section{Discussion}

This study was performed to evaluate the systemic absorption of cocaine after administration of atomised modified Moffett's solution prior to endoscopic sinus surgery (ESS). Six patients demonstrated a peak plasma cocaine concentration at $120 \mathrm{mi}-$ nutes post-administration (range 13-30 $\mu \mathrm{g} / \mathrm{L}$ ) and five patients showed an earlier peak after 60 minutes (range 14-31 $\mu \mathrm{g} / \mathrm{L}$ ). A single patient demonstrated a peak at 240 minutes $(42 \mu \mathrm{g} / \mathrm{L})$ post-administration. The mean peak plasma concentration at the most common time to peak (120 minutes post administration) was $20.35 \mu \mathrm{g} / \mathrm{L}$. Taking all peak values into account, the

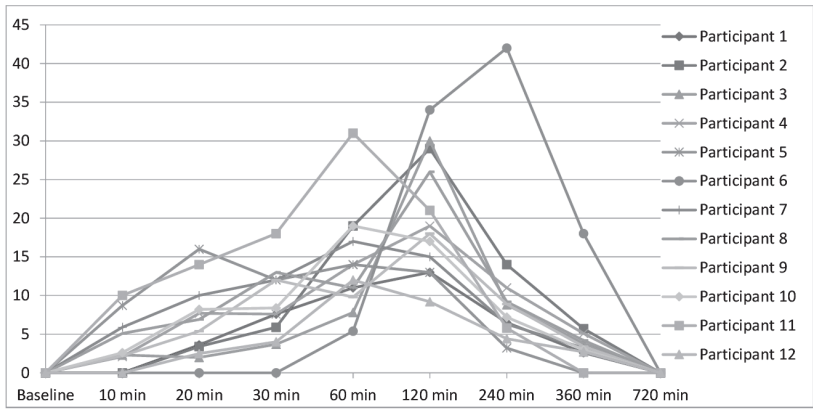

Figure 1. Plasma cocaine concentration $(\mu \mathrm{g} / \mathrm{L})$.

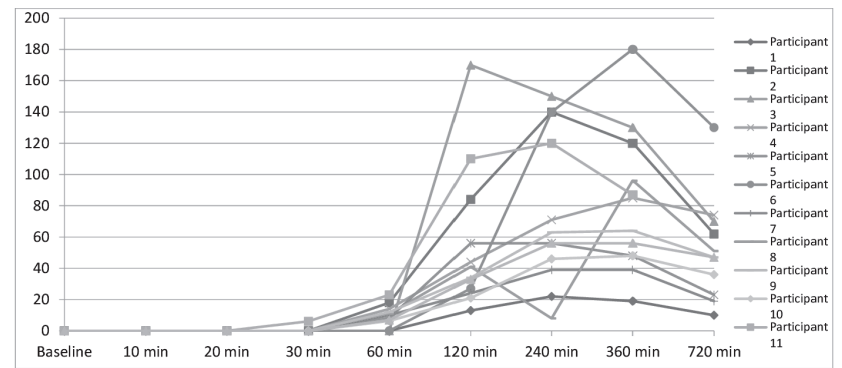

Figure 2. Plasma benzoylecgonine concentration ( $\mu \mathrm{g} / \mathrm{L})$.

mean was $22.6 \mu \mathrm{g} / \mathrm{L}$, a slightly higher concentration and likely clinically insignificant difference. We can probably assume that the true peak plasma level occurs somewhere between 60 and 120 minutes post-administration.

The mean peak plasma concentration of $20.35 \mu \mathrm{g} / \mathrm{L}$ is consistent with one other study by Quiney ${ }^{(6)}$. Four patients received intranasal Moffett's solution containing $200 \mathrm{mg}$ cocaine and $1 \mathrm{mg}$ adrenaline with $2 \mathrm{ml}$ 1\% sodium bicarbonate. The patients lay in the left lateral, right lateral and prone positions for 10 minutes each after application. The mean peak serum cocaine level was $18 \mu \mathrm{g} / \mathrm{L}$, occurring at 10 minutes. Interestingly, the peak level was similar to our study despite using twice the absolute amount of cocaine. Additionally, the peak occurred considerably earlier than in our study, at 10 minutes, which is likely secondary to the positional manoeuvres to maximise mucosal contact, and possibly the use of a sodium bicarbonate buffer which is known to increase the speed of absorption ${ }^{(3)}$. However, one might have expected a higher peak plasma concentration in this study.

It is also interesting to compare the peak level in our study to other studies using different cocaine amounts and application methods. In the study by Quiney, five patients also received $25 \%$ cocaine paste $(500 \mathrm{mg})$ applied to the nasal mucosa ${ }^{(6)}$. These patients demonstrated a peak of $50 \mu \mathrm{g} / \mathrm{L}$, which is consistent with the larger amount of cocaine being used. It was calculated that only approximately $5 \%$ of applied cocaine was absorbed. This is in contrast to studies by Greinwald et al and Liao et al. who demonstrated a mean absorption of approximately $35 \%$ of a $4 \mathrm{ml} 4 \%$ cocaine solution (160 mg) applied intranasally on cot- 
Table 2. Plasma cocaine concentration ( $\mu \mathrm{g} / \mathrm{L})$.

\begin{tabular}{|c|c|c|c|c|c|c|c|c|c|}
\hline Subject & Baseline & 10 mins & 20 mins & 30 mins & 60 mins & 2 hrs & 4 hrs & $6 \mathrm{hrs}$ & $12 \mathrm{hrs}$ \\
\hline 1 & 0 & 0 & 3.6 & 7.6 & 11 & 13 & 6.5 & 2.6 & 0 \\
\hline 2 & 0 & 0 & 3.4 & 5.9 & 19 & 29 & 14 & 5.7 & 0 \\
\hline 3 & 0 & 2.3 & 2 & 3.7 & 7.8 & 30 & 8.8 & 3.8 & 0 \\
\hline 4 & 0 & 2.1 & 7.7 & 7.6 & 14 & 19 & 11 & 5.1 & 0 \\
\hline 5 & 0 & 8.7 & 16 & 12 & 14 & 13 & 3.2 & 0 & 0 \\
\hline 6 & 0 & 0 & 0 & $\mathrm{n} / \mathrm{a}$ & 5.4 & 34 & 42 & 18 & 0 \\
\hline 7 & 0 & 5.9 & 10 & 12 & 17 & 15 & 6.3 & 3.2 & 0 \\
\hline 8 & 0 & 5.1 & 6.9 & 13 & 11 & 26 & 9.2 & 4.2 & 0 \\
\hline 9 & 0 & 2.2 & 5.4 & 12 & 9.8 & 18 & 8.9 & 3.4 & 0 \\
\hline 10 & 0 & 2.6 & 8.2 & 8.4 & 19 & 17 & 7.2 & 3 & 0 \\
\hline 11 & 0 & 10 & 14 & 18 & 31 & 21 & 5.8 & 0 & 0 \\
\hline 12 & 0 & 0 & 2.5 & 4 & 12 & 9.2 & 4.4 & 2.7 & 0 \\
\hline
\end{tabular}

Table 3. Mean plasma level ( $\mu \mathrm{g} / \mathrm{L})$.

\begin{tabular}{lccccccccc} 
& Baseline & $\mathbf{1 0 m i n s}$ & $\mathbf{2 0 m i n s}$ & $\mathbf{3 0 m i n s}$ & $\mathbf{6 0 m i n s}$ & $\mathbf{2 h r s}$ & $\mathbf{4 h r s}$ & $\mathbf{6 h r s}$ & $\mathbf{1 2 h r s}$ \\
\hline Cocaine & 0 & 3.24 & 6.64 & 9.47 & 14.25 & 20.35 & 10.61 & 4.31 & 0 \\
Benzoylecgonine & 0 & 0 & 0 & 0.55 & 9.12 & 54.75 & 75.92 & 81 & 51.73
\end{tabular}

Table 4. Plasma benzoylecgonine concentration ( $\mu \mathrm{g} / \mathrm{L})$.

\begin{tabular}{|c|c|c|c|c|c|c|c|c|c|}
\hline Subject & Baseline & 10 mins & 20 mins & 30 mins & 60 mins & 2 hrs & 4 hrs & $6 \mathrm{hrs}$ & 12 hrs \\
\hline 1 & 0 & 0 & 0 & 0 & 0 & 13 & 22 & 19 & 10 \\
\hline 2 & 0 & 0 & 0 & 0 & 18 & 84 & 140 & 120 & 62 \\
\hline 3 & 0 & 0 & 0 & 0 & 0 & 170 & 150 & 130 & 70 \\
\hline 4 & 0 & 0 & 0 & 0 & 14 & 44 & 71 & 85 & 74 \\
\hline 5 & 0 & 0 & 0 & 0 & 8.2 & 56 & 56 & 48 & 23 \\
\hline 6 & 0 & 0 & 0 & $n / a$ & 0 & 27 & 140 & 180 & 130 \\
\hline 7 & 0 & 0 & 0 & 0 & 10 & 24 & 39 & 39 & 19 \\
\hline 8 & 0 & 0 & 0 & 0 & 11 & 41 & 8 & 96 & 51 \\
\hline 9 & 0 & 0 & 0 & 0 & 12 & 34 & 63 & 64 & 47 \\
\hline 10 & 0 & 0 & 0 & 0 & 6.3 & 21 & 46 & 48 & 36 \\
\hline 11 & 0 & 0 & 0 & 6.1 & 23 & 110 & 120 & 87 & $\mathrm{n} / \mathrm{a}$ \\
\hline 12 & 0 & 0 & 0 & 0 & 6.9 & 33 & 56 & 56 & 47 \\
\hline
\end{tabular}

ton pledgets ${ }^{(4,5)}$. Liao et al. also demonstrated a linear relationship between cocaine dose and plasma level as well as mucosal contact time and plasma level ${ }^{(5)}$.

The peak absorption level in our study occurs later than expected at 120 minutes. Previous studies evaluating cocaine absorption have demonstrated earlier peak absorption, generally between 10-60 minutes ${ }^{(4,6,8,8)}$. Additionally, in our study, cocaine was still detectable in the plasma after six hours in the majority of patients (10 out of 12). A study by Van Dyke et al. also demon- strated measurable plasma cocaine levels up to $6 \mathrm{hrs}$ after a $10 \%$ cocaine solution (total dose $1.5 \mathrm{mg} / \mathrm{kg}$ ) was applied topically to the nasal mucosa prior to nasal intubation ${ }^{(7)}$. In that study, nasal swabs were taken three hours after application and were positive for cocaine, thus confirming the presence of cocaine locally on the mucosa for long periods after application ${ }^{(7)}$. It is likely our systemic profile represents prolonged absorption from the nasal mucosa, with the adrenaline in the modified Moffett's solution further increasing local vasoconstriction and preventing rapid absorption. 
Both Bromley et al. and Pfleiderer et al. confirm much lower systemic cocaine concentrations when adrenaline is added to the solution ${ }^{(8,9)}$. In addition, it is possible that despite removing excess from the nasopharynx at the commencement of surgery, a small amount of solution may have soaked the throat pack and led to a prolonged slow absorption.

The detection of cocaine within the blood up to six hours post surgery has further significance with the increasing rates of day case surgery seen within the UK and other centres worldwide ${ }^{(10)}$. Of note, in 2015 the United Kingdom introduced new laws governing legal levels for 8 illicit drugs whilst driving. A legal blood concentration of $10 \mu \mathrm{g} / \mathrm{L}$ for cocaine and $50 \mu \mathrm{g} / \mathrm{L}$ for benzoylecgonine were the established cut-offs; concentrations that could reliably not arise in the absence of substance consumption ${ }^{(11)}$. However, given the six hour systemic concentrations demonstrated in our research, it is theoretically possible a patient discharged within hours of ESS who undergoes drug testing will demonstrate a positive drug reading. Surgeons should thus counsel patients appropriately when eligible for day case ESS.

Despite the evidence that topical nasal cocaine is systemically absorbed, the average peak plasma concentration (most common time to peak of 120 minutes) of $20.35 \mu \mathrm{g} / \mathrm{L}$ in this study is low. We used $100 \mathrm{mg}$ cocaine per patient which is less than that used in several other studies and this is perhaps reflected in the lower peak concentration. With this dose we found the local vasoconstrictive effects to be excellent with a good surgical field in all patients. Using a lower dose helps to minimise the chance of any unwanted systemic side effects, as seen in the study by Liao et al. where hypertension and transient ventricular arrhythmia occurred only in those patients receiving higher doses of around $3 \mathrm{mg} / \mathrm{kg}^{(5)}$. Post mortem studies in cocaine related toxicity and death have shown variable plasma levels, Wetli reporting an average of $600 \mu \mathrm{g} / \mathrm{L}$ with a range of 100 to $900 \mu \mathrm{g} / \mathrm{L}^{(12)}$. It can be seen that our peak level of $20.35 \mu \mathrm{g} / \mathrm{L}$ is well below toxic doses. It is generally recommended amongst surgeons that the maximum safe topical cocaine dose is $3 \mathrm{mg} / \mathrm{kg}$, however the evidence for this recommendation is weak, largely based on these fatal doses in humans ${ }^{(13)}$. We support the lower maximum recommended dose in the UK is $1.5 \mathrm{mg} / \mathrm{kg}^{(14)}$.

Additional considerations when choosing the dose of cocaine are the addition of adrenaline, sodium bicarbonate buffer and the variations in absorption profiles that exist between patients. Several case reports have demonstrated the potential for increased systemic side effects, particularly cardiac, when cocaine is used with adrenaline ${ }^{(2)}$. This is perhaps reflective of the cumulative effect these drugs may have on increasing catecholamines. Additionally, the use of a sodium bicarbonate buffer, forming part of the traditional Moffett's solution, is still in practice in some centres. The higher $\mathrm{pH}$ of the solution allows an increased lipophilic proportion to rapidly diffuse across mucous membranes ${ }^{(3)}$. This allows for a quicker onset but may theoretically allow a higher peak serum level. Furthermore, as shown in our results, peak absorption of cocaine may be variable (range 12-42 $\mu \mathrm{g} / \mathrm{L}$ ) and likely reflects different mucosal contact patterns and time, local vascular factors and alterations in metabolism.

Apart from its systemic cardiac effects, cocaine has been postulated to have an impact on the depth of anaesthesia. Cocaine is a dopamine reuptake inhibitor, and recent work has suggested that the effect of awakening from anaesthesia may be due to activation of the dopamine 1 receptor, particularly in the ventral tegmental area ${ }^{(15,16)}$. It is possible that systemically absorbed cocaine may alter the depth of anaesthesia, however given the low concentrations seen in our research this is unlikely. In a previous study, plasma cocaine concentrations were measured on awake volunteers who had been administered either nasal or oral cocaine. Levels were correlated with a self-rating of how high each participant felt on a 6 point "high" scale. When the plasma concentration of cocaine averaged $54 \mu \mathrm{g} / \mathrm{L}$, the average "high" was only 0.8 when cocaine was given orally. An average rating of 1.1 on the scale correlated with a concentration of 36 $\mu \mathrm{g} / \mathrm{L}$ when given nasally ${ }^{(17)}$. Thus, given that the peak mean concentration in our study was below those that cause even minimal subjective CNS effects, it is unlikely our atomised modified Moffett's solution had any unwanted CNS affects or impact on depth of anaesthesia.

Although our sample group is small, we feel it provides an adequate profile of systemic absorption with reproducible results. In order to minimise degradation of the cocaine plasma samples, the tubes were stored in $-20^{\circ} \mathrm{C}$ freezers for short periods and then $-80^{\circ} \mathrm{C}$ freezers for longer periods prior to processing. The subsequent stability of the plasma cocaine levels was also validated by several means. Firstly, cocaine samples were analysed twice, separated by 2 weeks in a $-20^{\circ} \mathrm{C}$ freezer. Identical concentrations were found in the two samples demonstrating no degradation when stored at $-20^{\circ} \mathrm{C}$. Furthermore, cocaine is broken down both in vivo and in vitro to benzoylecgonine. If sample degradation had occurred, we would expect to see benzoylecgonine levels raised in the earlier samples (eg 10, 20, 30 minutes) but this is only seen from 60 minutes onwards, apart from in one patient. This is an appropriate degradation profile and we can thus assume the concentrations measured are accurate.

Data is missing on cocaine absorption at 30 minutes for a single patient. This is unlikely to have any influence on the overall results. Cocaine concentrations were reported at particular time intervals chosen to best capture the cocaine absorption profile. However, there is a slight possibility that between measured 
time intervals the plasma level had risen higher and subsequently fallen. If so this is likely to only be fractionally higher and not clinically relevant. Furthermore, the range in both age (25-79 years) and BMI (21-34 kg/m²) of our cohort may have led to some variations in systemic metabolism but there was no recorded renal or liver disease in any patient. Two patients reported occasional use of topical decongestant sprays but the short action of these sprays mean it is unlikely to have affected the results. Subject 6 reported intermittent prednisolone use and it is possible that if taken prior to surgery this may reduce local inflammation and subsequent blood flow leading to slower absorption of topical cocaine. This may explain the measured later peak at $\mathbf{2 4 0}$ minutes in this subject, however it is unclear why there was an overall higher systemic cocaine concentration seen in this patient. Overall, it is unlikely that concomitant medications played a significant role in altering systemic absorption.

\section{Conclusion}

This study demonstrates that there are low levels of systemic absorption of cocaine when administered in an atomised mo- dified Moffett's formulation prior to endoscopic sinus surgery. The peak absorption may occur up to 60-120 minutes following administration and there may subsequently be cocaine detected up to six hours post-administration. In addition, cocaine's metabolite, benzoylecgonine, may be detected up to 12 hours post administration. Understanding this systemic profile and its legal implications are important when counselling patients undergoing day case endoscopic sinus surgery.

\section{Acknowledgements}

ENT Technologies for funding contribution. Chris Kostakis from Forensic Science, South Australia for methodology and analysis.

\section{Authorship contribution}

JR, CG: Conceptualisation; DP, JR, CG, MK, SM, DB: Data collection; DP, DB: Data analysis; DP, MK. DB: Manuscript preparation; JR, CG: Review of Manuscript.

\section{Conflict of interest}

No conflict of interest exists.

\section{References}

1. Zhoa YC, Psaltis AJ. Hemostasis in sinus surgery. Curr Opin Otolaryngol Head Neck Surg. 2016; 24: 26 - 30

2. Saif AM, Farboud A, Delfosse E, Pope L Adke M. Assessing the safety and efficacy of drugs used in preparing the nose for diagnostic and therapeutic procedures: a systematic review. Clin Otolaryngol. 2016;41(5):546-63.

3. Benjamin E, Wong DKK, Choa D. Moffett's solution: a review of the evidence and scientific basis for the topical preparation of the nose. Clin Otolaryngol. 2004; 29: 582-87.

4. Greinwald JH, Holtel MR. Absorption of Topical Cocaine in Rhinologic Procedures. Laryngoscope. 1996; 106: 1223-1225.

5. Liao BS, Hilsinger Jr. RL, Rasgon BM, Matsuoka K, Adour KK. A Preliminary Study of Cocaine Absorption From the Nasal Mucosa. Laryngoscope. 1999; 109: 98-102.

6. Quiney RE. Intranasal topical cocaine: Moffett's method or topical cocaine paste? J Laryngol Otol. 1986; 100: 279-283.

7. Van Dyke C, Barash PG, Jatlow P, Byck R Cocaine: Plasma Concentrations After Intranasal Application in Man. Science. 1976; 191: 859-861.

8. Bromley L, Hayward A. Cocaine absorption from the nasal mucosa. Anaesthesia. 1988;

\section{3: 356-58}

9. Pfleiderer AG, Brockbank M. Cocaine and adrenaline: a safe or necessary combination in the nose? A study to determine the effect of adrenaline on the absorption and adverse side effects of cocaine. Clin Otolaryngol. 1988; 13: 421-26.

10. Alderwick H, Robertson R, Appleby J, Dunn P, Maguire D. Better Value in the NHS: the role of changes in clinical practice. London: The King's Fund; 2015.

11. Department of Transport. Evaluation of the new drug driving legislation, one year after its introduction [Internet]. United Kingdom; 2017 [cited 2018 June 01]. Available from: https://www.gov.uk/government/publications/drug-driving-law-evaluation.

12. Wetli CV, Fishbain DA. Cocaine-induce psychosis and sudden death in recreational users. J Forensic Sci. 1985; 30(3):873-80

13. Anderton JM, Nassar WY. Topical cocaine and general anaesthesia: an investigation of the efficacy and side effects of cocaine on the nasal mucosae. Anaesthesia. 1975; 30: 809-17.

14. Martindale Pharma. Cocaine Hydrochloride Solution 10\% w/v [Internet]. United Kingdom; 2014 [cited 2018 June 15]. Available from: https://www.medicines.org. uk/emc/product/3692/smpc
15. Taylor NE, Chemali JJ, Brown EN, Solt K. Activation of D1 dopamine receptors induces emergence from isoflurane general anesthesia. Anesthesiology. 2013; 118(1): 30-39.

16. Solt K, Van Dort CJ, Chemali JJ, Taylor NE, Kenny JD, Brown EN. Electrical stimulation of the ventral tegmental area induces reanimation from general anesthesia. Anesthesiology. 2014; 121(2): 311-19.

17. Van Dyke C, Jatlow P, Ungerer J, Barash PG, Byck R. Oral cocaine: plasma concentrations and central effects. Science. 1978; 200(4338): 211-3.

Dean Page

33 Bowen St

Prahran

Australia 3181

Tel: +61-43-416 0224

E-mail: deanejpage@gmail.com 